\title{
Peso ao nascer exerce influência sobre as aminotransferases e saúde de escolares com obesidade após uma intervenção?
}

http://dx.doi.org/10.11606/1807-5509201900020207

\author{
Micheli Lüttjohann DURÉ* \\ Rodrigo Machado ARDENGHI** \\ Cézane Priscila REUTER*** \\ Miria Suzana BURGOS $*, * * *$ \\ *Promoção da Saúde, \\ Universidade de Santa \\ Cruz do Sul, Santa \\ Cruz do Sul, RS, Brasil. \\ ${ }^{* *}$ Departamento de \\ Biologia e Farmácia \\ Universidade de Santa \\ Cruz do Sul, Santa \\ Cruz do Sul, RS, Brasi \\ ***Departamento de \\ Educação Física e \\ Saúde, Universidade \\ de Santa Cruz do Sul, \\ Santa Cruz do Sul, RS, \\ Brasil.
}

\section{Resumo}

A causa do sedentarismo, obesidade e esteatose hepática não alcóolica pode estar nos primeiros anos de vida ou, até mesmo, no peso de nascimento. Desta forma, objetivamos verificar se o peso ao nascer exerce influência sobre aminotransferases e saúde geral de escolares submetidos a uma intervenção. A amostra compõe-se de 39 escolares com sobrepeso e obesidade (19 no grupo controle e 20 no experimental), classificados com baixo peso, peso adequado e excesso de peso ao nascer. Foram avaliados: índice de massa corporal (IMC), aptidão cardiorrespiratória (APCR), flexibilidade, força abdominal, força de membros inferiores e superiores, agilidade, velocidade, aspartato aminotransferase (AST) e alanina aminotransferase (ALT). Foi encontrada diferença significativa, após a intervenção, do grupo experimental sobre o controle na flexibilidade ( $p=0,032$ no grupo peso adequado ao nascer), e na agilidade ( $p=0,035$ no grupo excesso de peso ao nascer). Observamos, após a intervenção, melhoras nas variáveis flexibilidade $(p=0,024, \Delta=5,88$ $\mathrm{cm}$ para o grupo baixo peso), força abdominal ( $p=0,013, \Delta=8,00$ repetições para grupo peso adequado ao nascer e $p=0,030, \Delta=5,00$ repetições para o grupo excesso de peso ao nascer), agilidade $(p=0,011$, $\Delta=-1,62$ segundos no grupo baixo peso/peso insuficiente ao nascer, $\mathrm{p}<0,001, \Delta=-1,25$ segundos para peso adequado ao nascer e $p=0,001, \Delta=-1,74$ segundos para grupo excesso de peso ao nascer) e força de membros inferiores $(p=0,002, \Delta=0,16 \mathrm{~cm}$ para o grupo excesso de peso). ALT teve uma piora no peso adequado ( $p=0,018, \Delta=5,24 \mathrm{UI} / \mathrm{L}$ ). Concluímos que a variável peso ao nascer não foi determinante para alteração dos níveis de aminotransferases, desempenho motor e de saúde após um programa de intervenção interdisciplinar.

PalavRas-Chave: Indicadores de Saúde; Desempenho Motor; Alanina Aminotransferase; Aspartato Aminotransferase; Obesidade.

\section{Introdução}

Uma das explicaçóes para o crescente aumento de doenças cardiovasculares (DCV), diabetes e valores elevados para alanina aminotransferase (ALT) e aspartato aminotransferase (AST), indicadores para esteatose hepática não alcóolica (EHNA), remete-se ao crescimento paralelo da obesidade e do sedentarismo ${ }^{1}$. Desta forma, a primeira causa para ambos pode ser o peso ao nascer ${ }^{2-4}$. Um estudo de prevalência aponta que $49 \%$ dos meninos e $67 \%$ das meninas de 10 a 12 anos da cidade de Pelotas, RS, Brasil, são considerados sedentários ${ }^{5}$. Outro estudo, também realizado no Brasil, assinala que cerca de 30\% dos escolares brasileiros estáo com excesso de peso ${ }^{6}$. 
Dentre os achados de DodD et al. ${ }^{7}$, gestantes com sobrepeso ou obesidade que melhoraram sua qualidade de vida, alimentação e exercício físico, durante a gestação, tendem a ter filhos com menos de $4,5 \mathrm{~kg}$; consequentemente, estas crianças estâo menos expostas a complicações, como a respiratória ${ }^{7}$. Além disso, estudos importantes remetem ao risco de uma criança que foi gerada de uma mãe com sobrepeso ou obesidade e que ganhou muito peso durante a gestação, nasce com alto peso e tende ao sobrepeso, tanto na adolescência, quanto na vida adulta ${ }^{8,9}$.

Já, por outro lado, em um estudo de revisão, mães mais jovens (<20 anos) e mais velhas ( $>$ de 35 anos), tendem a ter filhos com baixo peso ao nascer ${ }^{10}$. Observou-se, em outros estudos, que crianças que nasciam com baixo peso extremo e idade gestacional baixa, normalmente ganhavam muito peso durante o primeiro ano de vida e sendo assim, a prevalência para EHNA já na adolescência era alta, detectada através de análise das aminotransferases (AST e ALT) ${ }^{11,12}$. Neste sentido, pesquisadores acreditam que o sedentarismo, assim como da obesidade, pode se justificar nos primeiros anos de vida ${ }^{13,14}$ ou, até mesmo, no peso de nascimento da criança $a^{14}$. Algumas pesquisas nos mostram que crianças que nascem com baixo peso extremo tendem a ter problemas de coordenação motora ${ }^{15}$.
Outros estudos vão mais além, demonstrando que estas crianças estão mais propensas ao sedentarismo e, consequentemente, a baixos níveis de aptidão cardiorrespiratória (APCR) ${ }^{16}$.

Estudo australiano que objetivou verificar o desempenho motor e níveis de APCR em adolescentes que haviam nascido com baixo peso extremo, constatou déficit no desempenho motor, além disso, verificaram a relaçáo entre baixo desempenho motor e baixos níveis de APCR, demonstrando que problemas relacionados à coordenação motora podem ser preditores para níveis baixos de $\mathrm{APCR}^{2}$. Outros estudos indicam que os problemas de coordenaçáo motora tendem a persistir ${ }^{4}$, assim como os baixos níveis de $\mathrm{APCR}^{3,16}$. Já, em estudo de coorte, realizado em Pelotas, foi observada relaçáo inversa entre o baixo peso ao nascer em meninas e o sedentarismo no período de lazer ${ }^{17}$. $\mathrm{O}$ mesmo grupo não encontrou relação em outro estudo realizado 5 . Observou-se uma lacuna quando procuramos estudos que envolvam uma intervenção com exercícios físicos. Procurando elucidar a relação entre o peso ao nascer, indicadores de saúde e desempenho motor, este estudo tem como objetivo verificar se o peso ao nascer exerce influência sobre aminotransferases e saúde de escolares submetidos a um programa de intervenção interdisciplinar.

\section{Método}

Amostra: Este estudo quase-experimental é fruto de uma pesquisa realizada na Universidade de Santa Cruz do Sul, na qual se verificou a prevalência de crianças com sobrepeso e obesidade em escolas urbanas e rurais da cidade de Santa Cruz do Sul-RS. Além disso, está inserido no Projeto Saúde dos Escolares, realizado na Universidade de Santa Cruz do Sul (UNISC). Primeiramente, identificou-se, através dos dados obtidos em pesquisas anteriores, as escolas com maior índice de crianças e adolescentes com sobrepeso e obesidade para a realização de uma intervençáo com exercício físico e orientação nutricional. A amostra foi selecionada por conveniência, nas três escolas onde encontramos a maior prevalência de escolares com sobrepeso e obesidade. Desta forma, foram convidados a participar todos os escolares do sexo masculino e feminino com idades entre 7 a 17 anos com percentil superior a 85 e aptos à prática de exercício físico. Os escolares foram divididos em grupos controle e experimental. A escola que compóe o grupo controle foi definida por proximidade da Universidade. Por sua vez, cada grupo foi dividido em: peso insuficiente ao nascer, peso adequado e excesso de peso ao nascer. $O$ grupo experimental foi composto por 20 crianças e adolescentes de uma das escolas, e o grupo controle por 19 crianças e adolescentes das outras duas escolas, considerando a semelhança entre as características tanto de prevalência de sobrepeso e obesidade, como em relação às características do ambiente da escola em que a mesma está situada. Para a identificação das possíveis alteraçôes causadas pelo programa de intervenção realizou-se um cálculo amostral, considerando as variáveis em estudo, assim como um nível de significância de 95\%, um poder de teste de 0,80 e um efeito de 0,30 . Todos tiveram o termo de consentimento livre e esclarecido (TCLE) assinado e não tinham contraindicação para coleta sanguínea. Além disso, para completar o estudo, deveriam cumprir, no mínimo, $85 \%$ de aproveitamento das intervenções. 
Avaliações: As avaliações pré e pós-intervenção, de todos escolares, foram realizadas na Universidade de Santa Cruz do Sul.

Peso ao nascer: Obtido através de questionário e classificada de acordo com Puffer e Serrano: baixo peso: menor que $2.500 \mathrm{~g}$, peso insuficiente: 2.500 a $2.999 \mathrm{~g}$, peso adequado: $3.000 \mathrm{~g}$ a $3.999 \mathrm{~g}$ e excesso de peso: $4.000 \mathrm{~g}$ ou mais ${ }^{10}$.

Aptidão Cardiorrespiratória (APCR): Realizouse o teste de corrida/caminhada de 6 minutos (PROESP-2012), para o qual os escolares foram orientados a correr a maior parte do tempo possível, com o resultado obtido em metros ${ }^{18}$.

Flexibilidade: "Sentar e alcançar" com banco de Wells; neste teste, o indivíduo ficou sentado $\mathrm{com}$ as pernas estendidas, sendo anotado $(\mathrm{em} \mathrm{cm})$ o ponto máximo atingido pelas mãos da criança (sem flexionar os joelhos) ${ }^{18}$.

Força Abdominal: "Sit-up", no qual o sujeito realizou o número máximo de abdominais em 1 minuto ${ }^{18}$.

Força de Membros Superiores (MS): Arremesso de medicineball, no qual o avaliado deveria estar sentado e encostado numa parede com as pernas estendidas. $\mathrm{O}$ valor da distância em que a bola foi arremessada foi anotado em centímetros ${ }^{18}$.

Força de Membros Inferiores (MI): Salto horizontal, em que o escolar executou o salto com as duas pernas juntas, podendo flexionar os joelhos. Os resultados foram obtidos em centímetros ${ }^{18}$.

Agilidade: Teste do quadrado, no qual foram colocados quatro cones, afastados 4 metros um do outro, formando um quadrado. Ao sinal do avaliador, o escolar percorreu o quadrado através de diagonais no menor tempo possível ${ }^{18}$.

Velocidade: Corrida de 20 metros, a qual o escolar deveria percorrer no menor tempo possível, em segundos e centésimos ${ }^{18}$.
Análises Bioquimicas dos marcadores hepáticos: Aspartato aminotransferase (AST) e alanina aminotransferase (ALT), analisados em amostras de soro, coletados após 12 horas de jejum, através do Miura One (I.S.E., Rome, Italy), utilizando kits comerciais DiaSys (DiaSys Diagnostic Systems, Germany).

Intervenção: Realizou-se sessôes de exercícios físicos e orientação nutricional aos escolares por dezesseis semanas consecutivas, três vezes por semana, com duraçáo de cada sessão de 1 h e 30 minutos. Os exercícios foram realizados no ginásio da escola e monitorados por profissionais de Educação Física. A intensidade foi monitorada pela frequência cardíaca, estabelecendo-se uma zona alvo (40 a $80 \%$ da FC reserva); controlada através de um frequencímetro da marca Polar, modelo FT1 e estimada pela equação: FCreserva: $\mathrm{FC}_{\max }-$ FCrepouso, indicada por Villares, Ribeiro e Silva ${ }^{19}$. Ao final de cada sessão salientou-se a importância dos cuidados com alimentação através de explanaçóes de uma nutricionista.

A análise estatística foi desenvolvida no Programa Estatístico SPSS, versão 20.0 (IBM, Armonk, NY, USA). Testou-se a normalidade dos dados através do teste Shapiro-Wilk. Foram utilizados os testes paramétricos (teste t para amostras independentes e teste $\mathrm{t}$ pareado) e testes não paramétricos (MannWhitney e Wilcoxon). As diferenças significativas foram consideradas para $\mathrm{p}<0,05$. As medidas de tamanho de efeito foram calculadas conforme indicado por Sullivan e Feinn, por meio dos valores do teste $d$ de CoHEN $^{20}$.

Este estudo foi aprovado pelo Comitê de Ética da Universidade de Santa Cruz do Sul, sob o número CAAE:19620913.4.0000.5343, Parecer $n^{\circ}: 357.403$

QUADRO 1 - Intervenção

\begin{tabular}{|c|c|c|}
\hline Período & Objetivo e especificaçáo & Rotinas de intervençáo \\
\hline \multirow{5}{*}{$1^{\circ}$ encontro } & \multirow{5}{*}{$\begin{array}{l}\text { Adaptação ao estilo da intervenção } \\
\text { que se dará através de explicação } \\
\text { e demonstração das principais } \\
\text { atividades a serem realizadas; } \\
\text { Apresentação dos materiais utilizados; } \\
\text { Introdução do estilo de vida saudável. }\end{array}$} & $\begin{array}{l}\text { Apresentação dos profissionais, materiais e técnicas a } \\
\text { serem utilizadas; }\end{array}$ \\
\hline & & Atividades recreativas; \\
\hline & & Alongamento; \\
\hline & & Volta à calma; \\
\hline & & Orientação para os próximos encontros. \\
\hline
\end{tabular}

Continua 
Continuação

QUADRO 1 - Intervenção

\begin{tabular}{|c|c|c|}
\hline Período & Objetivo e especificação & Rotinas de intervenção \\
\hline \multirow{4}{*}{$\begin{array}{l}2^{\circ} \text { ao } 12^{\circ} \\
\text { encontro }\end{array}$} & \multirow{4}{*}{$\begin{array}{l}\text { Exercícios físicos de intensidade } \\
\text { moderada. Último dia de intervenção } \\
\text { desta semana será readaptada a zona } \\
\text { alvo para frequência cardíaca. }\end{array}$} & 10 min de aquecimento; \\
\hline & & $\begin{array}{l}35 \text { min de exercício aeróbico com características lúdicas } \\
\text { e desportivas (moderado a intenso); }\end{array}$ \\
\hline & & $\begin{array}{l}30 \text { min de reeducação postural, alongamento e volta } \\
\text { calma; }\end{array}$ \\
\hline & & 15 min de explanaçóes de uma nutricionista. \\
\hline \multirow{4}{*}{$\begin{array}{l}13^{\circ} \text { ao } 47^{\circ} \\
\text { encontro }\end{array}$} & \multirow{4}{*}{$\begin{array}{l}\text { Exercícios físicos de intensidade } \\
\text { moderada a intensa. A cada } 2 \text { semanas } \\
\text { será readaptada a zona alvo para } \\
\text { frequência cardíaca. }\end{array}$} & 10 min de aquecimento; \\
\hline & & $\begin{array}{l}35 \text { min de exercício aeróbico com características lúdicas } \\
\text { e desportivas (moderado a intenso); }\end{array}$ \\
\hline & & 30 min de reeducação postural, alongamento e volta calma; \\
\hline & & 15 min de explanaçóes de uma nutricionista. \\
\hline \multirow{4}{*}{$\begin{array}{l}48^{\circ} \\
\text { encontro }\end{array}$} & \multirow{4}{*}{$\begin{array}{l}\text { Encerramento com troca de } \\
\text { experiência entre os participantes } \\
\text { e confraternização com lanches } \\
\text { saudáveis }\end{array}$} & Aquecimento; \\
\hline & & Jogos recreativos; \\
\hline & & Alongamento; \\
\hline & & Confraternização. \\
\hline
\end{tabular}

\section{Resultados}

Observou-se que 9 escolares foram classificados como baixo peso/peso insuficiente ao nascer (grupo controle $=5$ e grupo experimental $=4$ ), 19 escolares classificados em peso adequado ao nascer (grupo controle $=12$ e grupo experimental $=7$ ) e 11 escolares com excesso de peso ao nascer (grupo controle $=2$ e experimental $=9$ ). Desta forma, 20 , dos 39 escolares, não nasceram com o peso adequado. Verificamos que houve diferença significativa, após a intervenção, do grupo experimental sobre o grupo controle, na flexibilidade $(\mathrm{p}=0,032$ e tamanho de efeito 1,150 no grupo peso adequado ao nascer) e na agilidade ( $\mathrm{p}=0,035$ e tamanho de efeito 2,550 no grupo excesso de peso). O tamanho de efeito ainda foi considerável na APCR $(1,010)$ e velocidade $(1,560)$ no grupo peso adequado ao nascer, bem como para força de membros superiores $(1,750)$ no grupo excesso de peso. Entretanto, houve diferença significativa do grupo controle sobre o experimental na velocidade ( $\mathrm{p}=0,003)$ (TABELA 1$)$.

Observamos, também, que não houve diferença significativa, no pré-teste e no pós-teste, entre os grupos: baixo peso, peso adequado e excesso de peso ao nascer. Entretanto, observamos, após a intervenção no grupo experimental, melhora nas variáveis flexibilidade ( $\mathrm{p}=0,024, \Delta=5,88$ para o grupo baixo peso/peso insuficiente ao nascer), força abdominal
( $\mathrm{p}=0,013, \Delta=8$ para grupo peso adequado ao nascer e p $=0,030, \Delta=5$, para o grupo excesso de peso ao nascer), agilidade ( $\mathrm{p}=0,011, \Delta=-1,62$ no grupo baixo peso/peso insuficiente ao nascer, $\mathrm{p}<0,001$, $\Delta=-1,25$ para peso adequado ao nascer e $\mathrm{p}=0,001$, $\Delta=-1,74$ para grupo excesso de peso ao nascer). Entretanto, observamos piora para a variável ALT ( $p=0,018, \Delta=5,24$ no grupo peso adequado ao nascer). AST teve uma melhora em todos os grupos, entretanto não foi significativa. Já, para o grupo controle, houve uma melhora significativa na agilidade ( $\mathrm{p}=0,005, \Delta=-0,58$ no grupo peso adequado ao nascer) e uma piora significativa na variável ALT ( $\mathrm{p}=0,043, \Delta=15,74$ no grupo baixo peso/peso insuficiente ao nascer, $\mathrm{p}=0,008, \Delta=5,24$ no grupo peso adequado ao nascer). $\mathrm{O}$ tamanho de efeito se mostra considerável no grupo controle nas variáveis: ALT $(2,432)$ para peso insuficiente; ALT $(1,385)$ para peso adequado; força de MS $(1,387)$, velocidade $(1,692)$ e ALT $(3,813)$ para excesso de peso. Para o grupo experimental, o tamanho de efeito se mostrou considerável na APCR $(1,573)$, flexibilidade $(1,121)$, força abdominal $(1,550)$, força de MI $(1,976)$ e ALT $(1,614)$ para baixo peso, bem como para os níveis de flexibilidade $(1,133)$, abdominal $(1,667)$, velocidade $(0,945)$ e ALT $(1,558)$ para o grupo peso adequado (TABELA 2). 
TABELA 1 - Indicadores de aptidão física relacionada à saúde e ao desempenho motor antes e após 4 meses de intervenção, estratificado pelo peso ao nascer

\begin{tabular}{|c|c|c|c|c|c|c|c|}
\hline \multirow{3}{*}{ Peso ao nascer } & \multicolumn{2}{|c|}{ Pré-teste } & \multirow{4}{*}{$\mathbf{p}$} & \multicolumn{2}{|c|}{ Pós-teste } & \multirow{4}{*}{$\mathbf{p}$} & \multirow{4}{*}{ Efeito* } \\
\hline & Grupo & & & Grupo & & & \\
\hline & Controle & Experimental & & Controle & Experimental & & \\
\hline & $\begin{array}{l}\text { Média } \\
\text { (DP) }\end{array}$ & $\begin{array}{l}\text { Média } \\
\text { (DP) }\end{array}$ & & $\begin{array}{l}\text { Média } \\
\text { (DP) }\end{array}$ & $\begin{array}{l}\text { Média } \\
\text { (DP) }\end{array}$ & & \\
\hline $\begin{array}{l}\text { Baixo peso/ } \\
\text { peso insuficiente }\end{array}$ & $\mathrm{N}=5$ & $\mathrm{~N}=4$ & & $\mathrm{~N}=5$ & $\mathrm{~N}=4$ & & \\
\hline \multicolumn{8}{|l|}{ Indicadores de saúde } \\
\hline $\operatorname{APCR}(\mathrm{m})$ & $\begin{array}{c}748,80 \\
(225,52)\end{array}$ & $\begin{array}{l}863,00 \\
(36,09)\end{array}$ & 0,190 & $\begin{array}{c}849,00 \\
(312,43)\end{array}$ & $\begin{array}{c}965,75 \\
(203,32)\end{array}$ & 0,541 & 0,440 \\
\hline Flexibilidade $(\mathrm{cm})$ & $\begin{array}{l}17,40 \\
(7,20)\end{array}$ & $\begin{array}{l}15,63 \\
(6,76)\end{array}$ & 0,717 & $\begin{array}{l}19,50 \\
(5,77)\end{array}$ & $\begin{array}{l}21,50 \\
(5,28)\end{array}$ & 0,608 & 0,360 \\
\hline Abdominal (rep) & $\begin{array}{c}21 \\
(11)\end{array}$ & $\begin{array}{l}20 \\
(8)\end{array}$ & 0,824 & $\begin{array}{l}23 \\
(8)\end{array}$ & $\begin{array}{c}22 \\
(11)\end{array}$ & 0,900 & 0,010 \\
\hline \multicolumn{8}{|l|}{ Desempenho motor } \\
\hline Força de MS (m) & $\begin{array}{c}3,37 \\
(0,99)\end{array}$ & $\begin{array}{c}2,80 \\
(1,12)\end{array}$ & 0,444 & $\begin{array}{c}3,47 \\
(0,84)\end{array}$ & $\begin{array}{c}3,60 \\
(1,27)\end{array}$ & 0,862 & 0,120 \\
\hline Força de MI (m) & $\begin{array}{c}1,11 \\
(0,14)\end{array}$ & $\begin{array}{c}1,10 \\
(0,12)\end{array}$ & 0,869 & $\begin{array}{c}1,18 \\
(0,21)\end{array}$ & $\begin{array}{l}1,25 \\
(0,21)\end{array}$ & 0,620 & 0,330 \\
\hline Agilidade (s) & $\begin{array}{l}7,70 \\
(1,13)\end{array}$ & $\begin{array}{c}8,72 \\
(0,88)\end{array}$ & 0,185 & $\begin{array}{l}7,47 \\
(0,85)\end{array}$ & $\begin{array}{l}7,10 \\
(1,03)\end{array}$ & 0,571 & 0,390 \\
\hline Velocidade (s) & $\begin{array}{l}5,23 \\
(0,58)\end{array}$ & $\begin{array}{l}4,95 \\
(0,59)\end{array}$ & 0,508 & $\begin{array}{l}5,04 \\
(0,65)\end{array}$ & $\begin{array}{l}5,17 \\
(0,43)\end{array}$ & 0,735 & 0,240 \\
\hline \multicolumn{8}{|l|}{ Marcadores hepáticos } \\
\hline ALT (UI/L) & $\begin{array}{l}10,46 \\
(1,63)\end{array}$ & $\begin{array}{l}12,00 \\
(1,64)\end{array}$ & 0,286 & $\begin{array}{c}26,20 \\
(24,22)\end{array}$ & $\begin{array}{l}16,88 \\
(1,56)\end{array}$ & 0,905 & 0,153 \\
\hline AST (UI/L) & $\begin{array}{l}20,96 \\
(4,28) \\
\end{array}$ & $\begin{array}{l}25,75 \\
(3,70) \\
\end{array}$ & 0,120 & $\begin{array}{l}23,78 \\
(4,69) \\
\end{array}$ & $\begin{array}{l}25,65 \\
(4,86) \\
\end{array}$ & 0,577 & 0,390 \\
\hline Peso adequado & $\mathrm{N}=12$ & $\mathrm{~N}=7$ & & $\mathrm{~N}=12$ & $\mathrm{~N}=7$ & & \\
\hline \multicolumn{8}{|l|}{ Indicadores de saúde } \\
\hline $\operatorname{APCR}(\mathrm{m})$ & $\begin{array}{l}773,75 \\
(119,93)\end{array}$ & $\begin{array}{c}805,71 \\
(121,22)\end{array}$ & 0,592 & $\begin{array}{c}882,42 \\
(204,70)\end{array}$ & $\begin{array}{c}689,14 \\
(175,31)\end{array}$ & 0,052 & 1,010 \\
\hline Flexibilidade $(\mathrm{cm})$ & $\begin{array}{l}18,08 \\
(7,80)\end{array}$ & $\begin{array}{l}21,57 \\
(4,94)\end{array}$ & 0,304 & $\begin{array}{l}19,08 \\
(6,80)\end{array}$ & $26,14(5,43)$ & 0,032 & 1,150 \\
\hline Abdominal (rep) & $\begin{array}{l}24 \\
(6)\end{array}$ & $\begin{array}{l}17 \\
(6)\end{array}$ & 0,014 & $\begin{array}{l}25 \\
(7)\end{array}$ & $\begin{array}{l}25 \\
(9)\end{array}$ & 0,869 & 0,000 \\
\hline \multicolumn{8}{|l|}{ Desempenho motor } \\
\hline Força de MS (m) & $\begin{array}{c}3,19 \\
(0,99)\end{array}$ & $\begin{array}{c}2,73 \\
(0,53)\end{array}$ & 0,273 & $\begin{array}{c}3,31 \\
(1,02)\end{array}$ & $\begin{array}{c}2,81 \\
(0,68)\end{array}$ & 0,271 & 0,580 \\
\hline Força de MI (m) & $\begin{array}{c}1,22 \\
(0,24)\end{array}$ & $\begin{array}{c}1,20 \\
(0,25)\end{array}$ & 0,879 & $\begin{array}{c}1,28 \\
(0,29)\end{array}$ & $\begin{array}{c}1,25 \\
(0,09)\end{array}$ & 0,826 & 0,140 \\
\hline Agilidade (s) & $\begin{array}{c}7,42 \\
(0,68)\end{array}$ & $\begin{array}{c}8,03 \\
(0,26)\end{array}$ & 0,038 & $\begin{array}{c}6,85 \\
(0,85)\end{array}$ & $\begin{array}{c}6,77 \\
(0,41)\end{array}$ & 0,836 & 0,120 \\
\hline Velocidade $(s)$ & $\begin{array}{c}4,62 \\
(0,48) \\
\end{array}$ & $\begin{array}{c}5,07 \\
(0,34) \\
\end{array}$ & 0,045 & $\begin{array}{c}4,68 \\
(0,44) \\
\end{array}$ & $\begin{array}{c}5,45 \\
(0,54) \\
\end{array}$ & 0,003 & 1,560 \\
\hline
\end{tabular}


APCR: aptidão cardiorrespiratória; MS: membros superiores;

Ml: membros inferiores;

ALT: alanina aminotransferase;

AST: aspartato aminotransferase; DP: desvio-padrão; teste t para amostras independentes ou Mann-Whitney; *

Cohen's d para comparação entre os grupos
Continuação

TABELA 1 - Indicadores de aptidão física relacionada à saúde e ao desempenho motor antes e após 4 meses de intervenção, estratificado pelo peso ao nascer

\begin{tabular}{|c|c|c|c|c|c|c|c|}
\hline \multirow{3}{*}{ Peso ao nascer } & \multicolumn{2}{|c|}{ Pré-teste } & \multirow{4}{*}{$\mathbf{p}$} & \multicolumn{2}{|c|}{ Pós-teste } & \multirow{4}{*}{$\mathbf{p}$} & \multirow{4}{*}{ Efeito* } \\
\hline & Grupo & Grupo & & Grupo & Grupo & & \\
\hline & Controle & Experimental & & Controle & Experimental & & \\
\hline & $\begin{array}{l}\text { Média } \\
\text { (DP) }\end{array}$ & $\begin{array}{c}\text { Média } \\
\text { (DP) }\end{array}$ & & $\begin{array}{l}\text { Média } \\
\text { (DP) }\end{array}$ & $\begin{array}{l}\text { Média } \\
\text { (DP) }\end{array}$ & & \\
\hline \multicolumn{8}{|l|}{ Marcadores hepáticos } \\
\hline ALT (UI/L) & $\begin{array}{l}11,38 \\
(3,13)\end{array}$ & $\begin{array}{l}12,07 \\
(2,69)\end{array}$ & 0,967 & $\begin{array}{l}16,80 \\
(5,06)\end{array}$ & $\begin{array}{l}17,31 \\
(3,79)\end{array}$ & 0,482 & 0,110 \\
\hline AST (UI/L) & $\begin{array}{l}25,95 \\
(5,78)\end{array}$ & $\begin{array}{l}26,41 \\
(3,92)\end{array}$ & 0,853 & $\begin{array}{l}25,05 \\
(5,66)\end{array}$ & $\begin{array}{l}24,71 \\
(3,45)\end{array}$ & 0,889 & 0,007 \\
\hline Excesso de peso & $\mathrm{N}=2$ & $\mathrm{~N}=9$ & & $\mathrm{~N}=2$ & $\mathrm{~N}=9$ & & \\
\hline \multicolumn{8}{|l|}{ Indicadores de saúde } \\
\hline APCR (m) & $\begin{array}{c}707,00 \\
(9,90)\end{array}$ & $\begin{array}{c}805,00 \\
(203,79)\end{array}$ & 0,727 & $\begin{array}{l}725,00 \\
(35,36)\end{array}$ & $\begin{array}{c}808,22 \\
(297,64)\end{array}$ & 0,713 & 0,390 \\
\hline Flexibilidade $(\mathrm{cm})$ & $\begin{array}{l}21,25 \\
(3,89)\end{array}$ & $\begin{array}{l}23,39 \\
(9,86)\end{array}$ & 0,777 & $\begin{array}{l}23,25 \\
(6,72)\end{array}$ & $\begin{array}{l}24,61 \\
(8,53)\end{array}$ & 0,869 & 0,180 \\
\hline Abdominal (rep) & $18(8)$ & $\begin{array}{l}19 \\
(8)\end{array}$ & 0,836 & $\begin{array}{c}17 \\
(10)\end{array}$ & $\begin{array}{c}24 \\
(10)\end{array}$ & 0,388 & 0,700 \\
\hline \multicolumn{8}{|l|}{ Desempenho motor } \\
\hline Força de MS (m) & $3,35(0,36)$ & $\begin{array}{c}2,75 \\
(0,80)\end{array}$ & 0,345 & $\begin{array}{c}3,88 \\
(0,18)\end{array}$ & $\begin{array}{c}2,84 \\
(0,82)\end{array}$ & 0,120 & 1,750 \\
\hline Força de MI (m) & $1,03(0,21)$ & $\begin{array}{c}1,08 \\
(0,24)\end{array}$ & 0,773 & $\begin{array}{c}1,17 \\
(0,30)\end{array}$ & $\begin{array}{c}1,25 \\
(0,26)\end{array}$ & 0,705 & 0,280 \\
\hline Agilidade (s) & $7,55(0,21)$ & $\begin{array}{c}8,53 \\
(0,84)\end{array}$ & 0,148 & $\begin{array}{l}7,84 \\
(0,12)\end{array}$ & $\begin{array}{c}6,79 \\
(0,57)\end{array}$ & 0,035 & 2,550 \\
\hline Velocidade (s) & $5,03(0,13)$ & $\begin{array}{c}5,19 \\
(0,56)\end{array}$ & 0,706 & $\begin{array}{c}5,25 \\
(0,20)\end{array}$ & $\begin{array}{c}5,49 \\
(0,65)\end{array}$ & 0,635 & 0,500 \\
\hline \multicolumn{8}{|l|}{ Marcadores hepáticos } \\
\hline ALT (UI/L) & $\begin{array}{l}11,25 \\
(1,06)\end{array}$ & $\begin{array}{l}12,84 \\
(5,31)\end{array}$ & 0,582 & $\begin{array}{l}18,25 \\
(3,46)\end{array}$ & $\begin{array}{l}15,71 \\
(5,58)\end{array}$ & 0,727 & 0,550 \\
\hline AST (UI/L) & $\begin{array}{l}21,35 \\
(3,75)\end{array}$ & $\begin{array}{l}28,90 \\
(7,60)\end{array}$ & 0,217 & $\begin{array}{l}21,50 \\
(7,50)\end{array}$ & $\begin{array}{l}27,70 \\
(8,42)\end{array}$ & 0,366 & 0,780 \\
\hline
\end{tabular}

TABELA 2 - Indicadores de aptidão física relacionada à saúde e ao desempenho motor antes e após 4 meses de intervenção, no grupo controle e experimental

\begin{tabular}{|c|c|c|c|c|c|c|c|c|c|c|}
\hline & \multicolumn{9}{|c|}{ Peso ao nascer } & \multirow[b]{2}{*}{$\mathbf{p}^{\S}$} \\
\hline & $\begin{array}{c}\text { Baixo } \\
\text { peso/peso } \\
\text { insuficiente }\end{array}$ & $\mathbf{p}$ & Efeito* & $\begin{array}{c}\text { Peso } \\
\text { adequado }\end{array}$ & $\mathbf{p}$ & Efeito* & $\begin{array}{l}\text { Excesso } \\
\text { de peso }\end{array}$ & $\mathbf{P}$ & Efeito* & \\
\hline Grupo Controle & $\mathrm{N}=5$ & & & $\mathrm{~N}=12$ & & & $\mathrm{~N}=2$ & & & \\
\hline \multicolumn{11}{|l|}{ Indicadores de saúde } \\
\hline$\triangle \mathrm{APCR}(\mathrm{m})$ & $\begin{array}{c}100,20 \\
(145,44)\end{array}$ & 0,225 & 0,429 & $\begin{array}{c}108,67 \\
(195,11)\end{array}$ & 0,077 & 0,552 & $\begin{array}{c}18,00 \\
(25,46)\end{array}$ & 0,317 & 0,553 & 0,491 \\
\hline$\Delta$ Flexibilidade $(\mathrm{cm})$ & $\begin{array}{c}2,10 \\
(5,85)\end{array}$ & 0,467 & 0,386 & $\begin{array}{c}1,00 \\
(4,95)\end{array}$ & 0,499 & 0,140 & $\begin{array}{c}2,00 \\
(2,83)\end{array}$ & 0,500 & 0,507 & 0,711 \\
\hline
\end{tabular}


TABELA 2 - Indicadores de aptidão física relacionada à saúde e ao desempenho motor antes e após 4 meses de intervenção, no grupo controle e experimental

\begin{tabular}{|c|c|c|c|c|c|c|c|c|c|c|}
\hline & \multicolumn{9}{|c|}{ Peso ao nascer } & \multirow[b]{2}{*}{$\mathbf{p}^{\S}$} \\
\hline & $\begin{array}{c}\text { Baixo } \\
\text { peso/peso } \\
\text { insuficiente }\end{array}$ & $\mathbf{p}$ & Efeito* & $\begin{array}{c}\text { Peso } \\
\text { adequado }\end{array}$ & $\mathbf{p}$ & Efeito* & $\begin{array}{l}\text { Excesso } \\
\text { de peso }\end{array}$ & $\mathbf{P}$ & Efeito* & \\
\hline$\Delta$ Abdominal (rep) & $\begin{array}{c}2 \\
(3)\end{array}$ & 0,294 & 0,479 & $\begin{array}{c}1 \\
(5)\end{array}$ & 0,318 & 0,208 & $\begin{array}{l}-1 \\
(2)\end{array}$ & 0,795 & $-0,125$ & 0,356 \\
\hline \multicolumn{11}{|l|}{ Desempenho motor } \\
\hline$\Delta$ Força de MS (m) & $\begin{array}{c}0,10 \\
(0,36)\end{array}$ & 0,560 & 0,149 & $\begin{array}{c}0,12 \\
(0,23)\end{array}$ & 0,106 & 0,369 & $\begin{array}{c}0,53 \\
(0,54)\end{array}$ & 0,396 & 1,387 & 0,723 \\
\hline$\Delta$ Força de MI (m) & $\begin{array}{c}0,07 \\
(0,10)\end{array}$ & 0,213 & 1,318 & $\begin{array}{c}0,06 \\
(0,23)\end{array}$ & 0,358 & 0,287 & $\begin{array}{c}0,14 \\
(0,09)\end{array}$ & 0,286 & 0,667 & 0,728 \\
\hline$\Delta$ Agilidade $(s)$ & $\begin{array}{l}-0,23 \\
(0,41)\end{array}$ & 0,278 & $-0,332$ & $\begin{array}{l}-0,58 \\
(0,57)\end{array}$ & 0,005 & $-1,024$ & $\begin{array}{c}0,29 \\
(0,08)\end{array}$ & 0,130 & 0.797 & 0,187 \\
\hline$\Delta$ Velocidade $(s)$ & $\begin{array}{l}-0,19 \\
(0,45)\end{array}$ & 0,397 & $-0,316$ & $\begin{array}{c}0,06 \\
(0,52)\end{array}$ & 0,699 & 0,106 & $\begin{array}{c}0,23 \\
(0,33)\end{array}$ & 0,514 & 1,692 & 0,210 \\
\hline \multicolumn{11}{|c|}{ Marcadores hepáticos } \\
\hline$\triangle \mathrm{ALT}(\mathrm{UI} / \mathrm{L})$ & $\begin{array}{c}15,74 \\
(23,00)\end{array}$ & 0,043 & 2,432 & $\begin{array}{c}5,24 \\
(3,50)\end{array}$ & 0,008 & 1,385 & $\begin{array}{c}2,87 \\
(6,54)\end{array}$ & 0,180 & 3,813 & 0,782 \\
\hline$\Delta \mathrm{AST}(\mathrm{UI} / \mathrm{L})$ & $\begin{array}{c}2,82 \\
(7,12) \\
\end{array}$ & 0,426 & 0,487 & $\begin{array}{l}-0,90 \\
(3,39) \\
\end{array}$ & 0,377 & $-0,196$ & $\begin{array}{c}0,15 \\
(3,75) \\
\end{array}$ & 0,964 & 0,023 & 0,688 \\
\hline $\begin{array}{l}\text { Grupo } \\
\text { Experimental }\end{array}$ & $\mathrm{N}=4$ & & & $\mathrm{~N}=7$ & & & $\mathrm{~N}=9$ & & & \\
\hline \multicolumn{11}{|c|}{ Indicadores de saúde } \\
\hline$\triangle \mathrm{APCR}(\mathrm{m})$ & $\begin{array}{c}102,75 \\
(193,72)\end{array}$ & 0,465 & 1,573 & $\begin{array}{l}-116,57 \\
(157,29)\end{array}$ & 0,128 & $-0,827$ & $\begin{array}{c}3,22 \\
(219,12)\end{array}$ & 0,678 & 0,016 & 0,167 \\
\hline$\Delta$ Flexibilidade $(\mathrm{cm})$ & $\begin{array}{c}5,88 \\
(2,75)\end{array}$ & 0,024 & 1,121 & $\begin{array}{c}4,57 \\
(5,59)\end{array}$ & 0,074 & 1.033 & $\begin{array}{c}1,22 \\
(6,12)\end{array}$ & 0,566 & 0,071 & 0,584 \\
\hline$\Delta$ Abdominal (rep) & $\begin{array}{c}2 \\
(4)\end{array}$ & 0,346 & 1,550 & $8(6)$ & 0,013 & 1,667 & $\begin{array}{l}5 \\
(6)\end{array}$ & 0,030 & 0,625 & 0,887 \\
\hline \multicolumn{11}{|l|}{ Desempenho motor } \\
\hline$\Delta$ Força de MS (m) & $\begin{array}{c}0,80 \\
(0,65)\end{array}$ & 0,090 & 0,799 & $\begin{array}{c}0,08 \\
(0,30)\end{array}$ & 0,495 & 0,459 & $\begin{array}{c}0,09 \\
(0,34)\end{array}$ & 0,472 & 0,112 & 0,310 \\
\hline$\Delta$ Força de MI (m) & $\begin{array}{c}0,16 \\
(0,14)\end{array}$ & 0,106 & 1,976 & $\begin{array}{c}0,06 \\
(0,25)\end{array}$ & 0,574 & 0,230 & $\begin{array}{c}0,16 \\
(0,11)\end{array}$ & 0,002 & 0,708 & 0,997 \\
\hline$\Delta$ Agilidade (s) & $\begin{array}{l}-1,62 \\
(0,57)\end{array}$ & 0,011 & $-1,841$ & $\begin{array}{l}-1,25 \\
(0,28)\end{array}$ & $<0,001$ & $-5,921$ & $\begin{array}{l}-1,74 \\
(0,94)\end{array}$ & 0,001 & $-1,647$ & 0,675 \\
\hline$\Delta$ Velocidade (s) & $\begin{array}{c}0,22 \\
(0,36)\end{array}$ & 0,310 & 0,373 & $\begin{array}{c}0,39 \\
(0,46)\end{array}$ & 0,067 & 0,945 & $\begin{array}{c}0,30 \\
(0,43)\end{array}$ & 0,069 & 0,733 & 0,650 \\
\hline \multicolumn{11}{|c|}{ Marcadores hepáticos } \\
\hline$\triangle \mathrm{ALT}(\mathrm{UI} / \mathrm{L})$ & $\begin{array}{c}4,88 \\
(2,86)\end{array}$ & 0,068 & 1,614 & $\begin{array}{c}5,24 \\
(3,50)\end{array}$ & 0,018 & 1,558 & $\begin{array}{c}2,87 \\
(6,54)\end{array}$ & 0,286 & 0,487 & 0,936 \\
\hline$\triangle \mathrm{AST}(\mathrm{UI} / \mathrm{L})$ & $\begin{array}{l}-0,10 \\
(3,70)\end{array}$ & 0,960 & $-0,020$ & $\begin{array}{l}-1,70 \\
(5,00)\end{array}$ & 0,403 & $-0,545$ & $\begin{array}{l}-1,20 \\
(8,36)\end{array}$ & 0,678 & $-0,144$ & 0,650 \\
\hline
\end{tabular}

APCR: aptidão cardiorrespiratória; MS: membros superiores; Ml: membros inferiores; ALT: alanina aminotransferase; AST: aspartato aminotransferase; $\Delta$ : diferença após a intervenção; dados expressos em média (desvio-padrão); teste t pareado ou Wilcoxon; svalor de $p$ na comparação dos três grupos (ANOVA ou Kruskal-Wallis); ${ }^{*}$ Cohen's d para medidas repetidas. 


\section{Discussão}

Nosso estudo demonstrou que mais da metade de nossos escolares com sobrepeso ou obesidade estavam com peso inadequado ao nascer. Nesta perspectiva, objetivamos verificar se o peso ao nascer exerce influência sobre transaminases, os indicadores de aptidão física e saúde em escolares submetidos a um programa de intervenção interdisciplinar. Observamos que esta não ocorre quando observamos os três grupos (baixo peso, peso ideal, excesso de peso ao nascer), corroborando os achados do grupo de Pelotas ${ }^{17}$. Um estudo realizado na Finlândia verificou que o peso ao nascer foi associado positivamente com a força muscular e condicionamento aeróbico aos 31 anos de idade, independentes do tamanho do corpo do adulto ${ }^{21}$. Outro fator importante encontrado na literatura nos mostra que adultos que nasceram com muito baixo peso tendem a serem menos ativos no seu tempo livre ${ }^{22}$, apontando a importância de termos um olhar mais atento a este grupo que possui uma tendência maior a doenças cardiovasculares ${ }^{23}$. Observamos em nosso estudo que, após a intervenção, os escolares que nasceram com baixo peso/peso insuficiente melhoraram na flexibilidade e agilidade, assim como APCR, velocidade e força de MS. Há ainda, autores que demonstram que o risco de nascer com baixo peso é o ganho acelerado de peso no primeiro ano de vida, tornando-os mais propensos a doenças cardiovasculares e EHNA ${ }^{11,12}$. Em estudo realizado com ratos Wistar, tanto o peso ao nascer, quanto a dieta pré-natal, interfere no perfil metabólico destes ratos na fase adulta, demonstrando a importância de promover um estilo de vida desde a vida intrauterina ${ }^{24}$. Observamos que, mesmo após a intervenção, todos escolares nascidos com peso adequado, pioraram significativamente os níveis de ALT. O mesmo ocorrendo no grupo controle; este último ainda piorou para o grupo de baixo peso/peso insuficiente ao nascer. Em um estudo com 1.754 crianças australianas, das quais $15,2 \%$ apresentaram EHNA, foi observada a relação da adiposidade com o aparecimento e com a gravidade de EHNA aos 17 anos de idade, apesar de que, náo se observou relação com peso ao nascer ${ }^{25}$. De mesma forma, em outro estudo, os autores não encontraram relaçáo do peso ao nascer com EHNA; entretanto, ganho acelerado de peso nos primeiros três meses de vida está associada a um maior risco de EHNA no início da idade adulta ${ }^{26}$. Também é observada esta relação do ganho acelerado de peso com $\mathrm{DCV}^{27}$. Em um estudo transversal com 100 adolescentes de 12 a 18 anos, ALT demonstrou relação inversa com $\mathrm{APCR}^{28}$. Desta forma, podemos admitir que primeira opção para tratamento deva ser dieta adequada e exercício físico, com perda de peso ${ }^{29}$. Em nosso estudo, no grupo peso adequado ao nascer, os escolares, após a intervenção, melhoraram na variável agilidade. Já, num estudo prospectivo longitudinal que trabalhou com amostra de gêmeos, observaram que, tanto as crianças que estavam com baixo peso, quanto às de alto peso, possuíam déficit no consumo de oxigênio na adolescência, entretanto, quando se ajustava ao IMC dos pais, estas diferenças deixavam de ser significativas ${ }^{30}$. Em nosso estudo, a APCR não se mostrou significativa embora o tamanho de efeito para o grupo de baixo peso tenha sido 1,573. Assim como devemos ressaltar o tamanho de efeito para este grupo na flexibilidade, abdominal, forca de membros inferiores e ALT. Para o excesso de peso ao nascer, observou-se, após a intervenção, melhoras significativas nos indicadores de saúde (força abdominal) e desempenho motor (força de $\mathrm{MI}$; consideramos que este pode ser o início para um estilo de vida mais ativo para estes escolares que, desde o nascimento, convivem com o excesso de peso.

Concluímos que a variável peso ao nascer não foi determinante para as aminotransferases, melhor desempenho motor e de saúde nesta pesquisa, apesar de terem sido observadas melhoras significativas após a intervenção.

\section{Abstract \\ Birth weight influences aminotransferases levels and health of children with obesity after an intervention?}

The cause of physical inactivity, obesity and non-alcoholic fatty liver disease may be early in life or even in birth weight. Thus, we aimed to determine whether birth weight influences aminotransferases and health of schoolchildren after an intervention. The sample consists of 39 children with overweight and obesity 
(19 in the control group and 20 in experimental), classified as underweight, normal weight and excess weight at birth. Were assessed: body mass index (BMI), cardiorespiratory fitness (CRF), flexibility, abdominal strength, strength of lower and upper limbs, agility, speed, aspartate aminotransferase (AST) and alanine aminotransferase (ALT). There was a significant difference after the intervention, in the experimental group over the control in flexibility ( $p=0.032$ in the normal weight at birth group) and agility ( $p=0.035$ in the excess weight at birth group). We observed, after the intervention, improvements in flexibility ( $p=0.024$, $\Delta=5.88 \mathrm{~cm}$ for underweight at birth group), abdominal strength ( $\mathrm{p}=0.013, \Delta=8.00$ repetitions for normal weight at birth group and $p=0.030, \Delta=5.00$ repetitions for the excess weight at birth group), agility ( $p=0.011, \Delta=-1.62$ seconds in the underweight at birth group, $p<0.001, \Delta=-1.25$ seconds for normal weight at birth and $p=0.001, \Delta=-1.74$ seconds to excess weight at birth) and strength of the lower limbs ( $p=0.002, \Delta=0.16 \mathrm{~cm}$ for excess weight group). ALT had a worsening in the normal weight ( $p=0.018$, $\Delta=5.24 \mathrm{UI} / \mathrm{L}$ ). We conclude that the variable birth weight was not decisive for change in aminotransferase levels, motor performance and health-related physical fitness after an interdisciplinary intervention program.

KeYwords: Health-related Physical Fitness; Motor Performance; Alanine Transaminase; Aspartate Aminotransferase; Obesity.

\section{Conflito de interesse}

Declaramos que não há conflito de interesses.

\section{Referências}

1. Han JC, Lawlor DA, Kimm SY. Childhood obesity. Lancet. 2010;375(9727):1737-48.

2. Burns YR, Danks M, O'Callaghan MJ, et al. Motor coordination difficulties and physical fitness of extremely-low-birthweight children. Dev Med Child Neurol. 2009;51(2):136-42.

3. Keller H, Ayub BV, Saigal S, Bar-Or O. Neuromotor ability in 5- to 7-year-old children with very low or extremely low birthweight. Dev Med Child Neurol. 1998;40(10):661-6.

4. Whitaker AH, Feldman JF, Lorenz JM, et al. Motor and cognitive outcomes in nondisabled low-birth-weight adolescents: early determinants. Arch Pediatr Adolesc Med. 2006;160(10):1040-6.

5. Hallal PC, Wells JC, Reichert FF, Anselmi L, Victora CG. Early determinants of physical activity in adolescence: prospective birth cohort study. BMJ. 2006;332(7548):1002-7.

6. Flores LS, Gaya AR, Petersen RD, Gaya A. Trends of underweight, overweight, and obesity in Brazilian children and adolescents. J Pediatr (Rio J). 2013;89(5):456-61.

7. Dodd JM, McPhee AJ, Turnbull D, et al. The effects of antenatal dietary and lifestyle advice for women who are overweight or obese on neonatal health outcomes: the LIMIT randomised trial. BMC Med. 2014 Oct 13;12(1):163.

8. Godfrey KM, Inskip HM, Hanson MA. The long-term effects of prenatal development on growth and metabolism. Semin Reprod Med. 2011;29(3):257-65.

9. Baird J, Fisher D, Lucas P, Kleijnen J, Roberts H, Law C. Being big or growing fast: systematic review of size and growth in infancy and later obesity. BMJ. 2005;331(7522):929.

10. Mariotoni GG, Barros Filho AA. Peso ao nascer e mortalidade hospitalar entre nascidos vivos, 1975-1996. Rev Saúde Pública. 2000;34(1):71-6.

11. Anderson EL, Howe LD, Fraser A, et al. Weight trajectories through infancy and childhood and risk of non-alcoholic fatty liver disease in adolescence: the ALSPAC study. J Hepatol. 2014;61(3):626-32.

12. Faienza MF, Brunetti G, Ventura A, et al. Nonalcoholic fatty liver disease in prepubertal children born small for gestational age: influence of rapid weight catch-up growth. Horm Res Paediatr. 2013;79(2):103-9.

13. Monteiro POA, Victora C. Rapid growth in infancy and childhood and obesity in later life-a systematic review. Obes Rev. 2005;6(2):143-54. 
14. van Deutekom AW, Chinapaw MJ, Vrijkotte TG, Gemke RJ. Study protocol: the relation of birth weight and infant growth trajectories with physical fitness, physical activity and sedentary behavior at 8-9 years of age-the ABCD study. BMC Pediatr. 2013;13(1):102.

15. Bracewell M, Marlow N. Patterns of motor disability in very preterm children. Ment Retard Dev Disabil Res Rev. 2002;8(4):241-8.

16. Rogers M, Fay TB, Whitfield MF, Tomlinson J, Grunau RE. Aerobic capacity, strength, flexibility, and activity level in unimpaired extremely low birth weight $(\leq 800 \mathrm{~g})$ survivors at 17 years of age compared with term-born control subjects. Pediatrics. 2005;116(1):e58-e65.

17. Azevedo MR, Horta BL, Gigante DP, Victora CG, Barros FC. [Factors associated to leisure-time sedentary lifestyle in adults of 1982 birth cohort, Pelotas, Southern Brazil]. Rev Saúde Pública. 2008;42(Suppl 2):70-7.

18. Gaya A, Lemos A, Gaya A, Teixeira D, Pinheiro E, Moreira R. Projeto Esporte Brasil PROESP-Br: manual de aplicaçáo de medidas e testes, normas e critérios de avaliação. Porto Alegre: UFRGS; 2007.

19. Villares SMF, Ribeiro MM, Silva AG. Obesidade infantil e exercício. Evid Obes. 2003;13(13):1-5.

20. Sullivan GM, Feinn R. Using Effect Size - or Why the P Value Is Not Enough. J Grad Med Educ. 2012;4(3):279-82.

21. Ridgway CL, Ong KK, Tammelin T, Sharp SJ, Ekelund U, Jarvelin MR. Birth size, infant weight gain, and motor development influence adult physical performance. Med Sci Sports Exerc. 2009;41(6):1212-21.

22. Kajantie E, Strang-Karlsson S, Hovi P, et al. Adults born at very low birth weight exercise less than their peers born at term. J Pediatr. 2010;157(4):610-6, 616.e1.

23. Carnethon MR, Gidding SS, Nehgme R, Sidney S, Jacobs Jr DR, Liu K. Cardiorespiratory fitness in young adulthood and the development of cardiovascular disease risk factors. JAMA. 2003;290(23):3092-100.

24. Eleftheriades M, Pervanidou P, Vafaei H, et al. Metabolic profiles of adult Wistar rats in relation to prenatal and postnatal nutritional manipulation: the role of birthweight. Hormones (Athens). 2014;13(2):268-79.

25. 25. Ayonrinde OT, Olynyk JK, Marsh JA, et al. Childhood adiposity trajectories and risk of nonalcoholic fatty liver disease in adolescents. J Gastroenterol Hepatol. 2015;30(1):163-71.

26. Breij LM, Kerkhof GF, Hokken-Koelega AC. Accelerated infant weight gain and risk for nonalcoholic fatty liver disease in early adulthood. J Clin Endocrinol Metab. 2014;99(4):1189-95.

27. Kerkhof GF, Willemsen RH, Leunissen RW, Breukhoven PE, Hokken-Koelega AC. Health profile of young adults born preterm: negative effects of rapid weight gain in early life. J Clin Endocrinol Metab. 2012;97(12):4498-506.

28. Kelishadi R, Cook SR, Amra B, Adibi A. Factors associated with insulin resistance and non-alcoholic fatty liver disease among youths. Atherosclerosis. 2009;204(2):538-43.

29. Nobili V, Alisi A, Raponi M. Pediatric non-alcoholic fatty liver disease: preventive and therapeutic value of lifestyle intervention. World J Gastroenterol. 2009;15(48):6017-22.

30. Touwslager RN, Gielen M, Tan FE, et al. Genetic, Maternal and Placental Factors in the Association between Birth Weight and Physical Fitness: a longitudinal twin study. PLoS One. 2013;8(10):e76423.

\begin{tabular}{r|r} 
ENDEREço & \\
Miria Suzana Burgos & \\
Avenida Independência, 2293 - bloco 42, sala & Recebido para publicação: 14/08/2015 \\
4206 - Universitário & Revisão: 21/12/2017 \\
$96815-900$ - Santa Cruz do Sul - RS - BRASIL & Aceito: 02/03/2018 \\
e-mail: michemburgos@unisc.br; & \\
michelild@yahoo.com.br & \\
\hline
\end{tabular}

